\title{
Cultural Basis of Metaphors Translation: Case of Emotions in Persian and English
}

\author{
Fatemeh Safarnejad $^{1}$, Imran Ho-Abdullah ${ }^{1} \&$ Norsimah Mat Awal ${ }^{1}$ \\ ${ }^{1}$ School of Language Studies and Linguistics, University Kebangsaan Malaysia (UKM), Bangi, Malaysia \\ Correspondence: Fatemeh Safarnejad, School of Language Studies and Linguistics, University Kebangsaan \\ Malaysia (UKM), Bangi 43600, Selangor, Malaysia. Tel: 60-17-618-4030. E-mail: safarnejad.a@gmail.com
}

Received: November 15, 2013 Accepted: February 12, 2014 Online Published: March 31, 2014

doi:10.5539/ass.v10n7p107

URL: http://dx.doi.org/10.5539/ass.v10n7p107

\begin{abstract}
Metaphorical expressions often involve culturally-specific concepts, embodying associations related to a particular cultural community. Metaphor translation poses the problems of switching between different cultural references, as well as conceptual and linguistic perspectives. Dealing with metaphors in translation, thus, is not simply a matter of identifying the linguistic correspondences in two languages under study, but of identifying correspondences between their conceptual systems corresponding to their different cultural models. The main purpose of this paper is to present the findings of a study that investigated emotive metaphoric conceptualizations and their dominant patterns in Persian and English. The emotions under study are metaphorical expressions of happiness and sadness which have been compiled from a literary source text and its two corresponding target texts. The Metaphor Identification Procedures (MIP), proposed by the Pragglejazz group (2007), and Lakoff and Johnson's (1980) Conceptual Metaphor Theory (CMT) were adopted as the framework for analysis. Our findings revealed that there are many cultural similarities and differences between emotive metaphorical concepts in Persian and English.
\end{abstract}

Keywords: metaphorical expressions, conceptual metaphor, translation patterns, cognitive analysis, sadness, happiness

\section{Introduction}

Translation from one language to another is not possible if there is no adequate knowledge of the two cultures involved. It is a well-known fact that the art of translation is not ordinarily changing one text to another or transposing words from one language to another. Rather, translation entails the act of transferring one entire culture to another. Since there is no one-to-one correspondence between two different languages, there may exist difficulties in translation from one language to another with regard to cultural differences such as translating from Persian into English and vice versa. The difference between Source Language (SL) and Target Language (TL) and other variations in cultures can sometimes pose challenges in the process of translation. One of the most challenging tasks a translator faces is the difficulty of translating metaphorical expressions to another language. Even translation scholars acknowledge this difficulty. Dagut (1976) pointed out that translation problem is represented by metaphor because it lacks universality thereby making it impossible to be translated word by word. He claimed that simple general rule is not in existence for metaphor translation but the translatability nature of any given SL metaphor should depend upon the particular cultural experiences and semantic associations which it should exploit. It could also be noticed that if the source and the target languages contain in common cultural experiences and the semantic associations, there is very likelihood that the target language can produce a metaphor. However, if the speakers of the TL do not share cultural experiences, there is less likelihood that a metaphor could be translated in a similar way. Some studies viewed the concept of culture as a situation where knowledge, proficiency and perception should be fundamentally translated while it was generally viewed that metaphor translation should also be particularized (Snell-Hornby,1988; Tabakowska, 1993). The differences in cultures have seen the experiences from different ways and that was the reason why metaphor is viewed as having specific culture. Some studies revealed that culture is relevant when it is playing role as a determinant factor in metaphor translation (Nida,1964; Catford, 1965; Snell-Hornby, 1988; Tabakowska, 1993). In addition to the scholars above, Dobrzynska (1995) claimed that the metaphorical interpretation is strongly culturally conditioned. Other scholars like Van den Broeck (1981) believed that the translatability nature 
of metaphor is inversely proportional to the degree of the specific cultural information that is included in the metaphor and the manner through which the information is structured in the text (Van den Broeck, 1981). However, it is justifiable to think that for translating a metaphor, a translator faces problems with the rules meant for metaphor translation that do not take into account cultural experience and semantic association. It would, therefore, be suggested that Dagut's (1987) argument is relevant to the present study because it supports the problem statement of the study that problems of translating metaphors do exist because they are not governed by any rule, and that there are no rules for translating metaphors.

The present study aimed to examine how emotive metaphorical expressions have been dealt with in two languages of Persian and English. Persian and English languages are naturally dissimilar since both have different cultural identities. Furthermore, language expressions could be seen as the main difference between Persian and English metaphoric expressions that in turn could be viewed as the factor enriching the societal cultural characteristics. Above all, there is a great cultural gap between Persian and English which makes the translation of emotive metaphorical expressions more difficult.

\section{Background of Study}

Translation scholars have attempted to identify translation correspondence. The studies conducted by Hiraga (1991), Mandelblit (1995), Schäffner (2004), Kovecses (2005), Al-Zoubi (2006), Al-Hasnawi (2007), Maalej (2008) and Iranmanesh and Kaur (2010) have viewed metaphor translation from a cognitive linguistic perspective through addressing either one or more aspects. These studies distinguished between similar mapping condition (SMC) and the different mapping condition (DMC) in the sense that the source language (SL) and the target language (TL) in the SMC case used the identical metaphor to conceptualize a particular notion while both SL and TL conceptualize a particular notion using a different metaphor in the DMC case. These approaches, which were largely descriptive, focused on how metaphors and metaphorical expressions were treated in actual translations. Al-Zoubi, (2006) declare that since there is a relationship between metaphors and different cultural domains, the translator ought to conduct the conceptual mapping for the TL reader in which the cognitive equivalence in the target culture has to be looked. The task of translation ought to be easier if the SL and the TL cultures conceptualize experience in a similar way (Al-Zoubi, 2006). In a similar study conducted by Al-hasnawi (2007), it was proposed that the cogno-cultural framework and the cognitive equivalence hypothesis used for translating metaphors based upon the cognitive translation hypothesis (CTH) proposed by Mandelblit (1995) should be used to distinguish between SMC and DMC. The source and target languages in SMC use the same metaphor to conceptualize a domain while the source and target languages in DMC use different metaphors. In an effort to modify Mandelblit's SMC and DMC, Al-hasnawi (2007) have suggested that there are three cognitive mapping conditions for translating metaphors. These three cognitive mapping conditions include:

1) Metaphors which have similar mapping conditions,

2) Metaphors which have similar mapping conditions but were differently lexically implemented, and

3) Metaphors which have different mapping conditions.

The above listed conditions are represented as continuum where similar mapping conditions at one end show languages and cultures greatly converging and sharing the universal metaphors that are grounded in a common experiential way. The greater divergence between languages shown at the other end of different mapping conditions is due to marked cultural differences. In a similar way, due to the cultural or ethical system of each language with different lexicalisations, they are placed at an intermediate stage demonstrating some differences in the mapping conditions. However, it has been shown that the first and second results in the conditions are equivalent to the TL metaphor or the TL simile. The third condition reveals that the translator opted for a TL simile, a footnote, paraphrase, explanatory remark or the metaphor is omitted whenever there is a failure in all the other options (Al-hasnawi, 2007).

In sum, all the cognitive approaches mentioned above to translate metaphors provide a view on the types of variation that can occur in translation. Reviewing the related literature brought to light the fact that the cognitive metaphor translation as a framework can help studies in the field of metaphor translation particularly in avoiding the confusion the term "metaphor" might cause being used to refer to both the cognitive and the linguistic phenomena. Consequently, the general assumption is that the cognitive strategy for metaphor translation should simplify the whole procedure based upon the fact it raises the awareness of the inventories of cross-domain mapping in SL and TL. This helps in retaining the metaphorical expressions contained in any type of text. Nonetheless, it has been shown that culture can play an important role in the transfer of conceptual metaphor from one conceptual system to another. Based upon the studies mentioned above, the study in hand proposes a combination of translation strategies that would serve as a point of departure for identifying patterns of 
translation. These methods would be probably reviewed and further refined in the light of metaphor analysis' findings.

\section{Theoretical Framework}

The domain of translation has so far been described and understood in association with different metaphors. Some of these metaphors are reflected in everyday language, some of them appear in historical texts about translation, and some have left their mark on contemporary translation studies. There are different theories and approaches regarding translation of metaphor, each of which has investigated this problem from different perspectives. The search for a clear and reliable conceptual framework for this study resulted in the construction of a framework based on the general framework of conceptual metaphor theory (CMT) with the metaphor identification procedures (MIP), proposed by the Pragglejazz group (2007). The data will be analyzed by means of these two theories. The following paragraphs will first discuss the theory of conceptual metaphors, and then explain the metaphor identification procedures (MIP) designed by the Pragglejaz group (2007) and elaborated by Steen et al. (2008). The new view of metaphor presented by Lakoff and Johnson (1980) is that it is a basic cognitive function that helps in comprehending the world and structuring abstract concepts. The human conceptual system is viewed in terms of both thinking and acting as it is seen as being fundamentally metaphorical in nature. This equally gave rise to the cognitive linguistic view of metaphor, which is also referred to as the contemporary theory of metaphor, through which metaphor is approached as a thought process; and a manner of experience organization and articulation (Lakoff, 1993). The conceptual metaphor could mean comprehending one of the conceptual domains in place of another conceptual domain and these are described as the source domain and target domain. However, the target domain is the domain which is more abstract in nature while the source domain is more physical in nature which could be employed for better understanding of the target domain. The conceptual metaphor could also be understood as the cross-domain mapping or a set of mappings or correspondences.

A new method was created by some metaphor scholars from various scholastic disciplines, known as the Pragglejaz Group, to recognize those words that were used as metaphor in written and spoken language. The method was called Metaphor Identification Process (MIP). This process attempts to ascertain whether use of each lexical element in a dialogue stretch can be portrayed as metaphor in a special framework. The rationale for this method is that a disparity between the contextual implications of a lexical element and its more fundamental connotation gives rise to metaphorical meaning. The fundamental connotation is missing from the real situation but is apparent in other contexts. A lexical unit is recognized as metaphorical if one is able to map between the basic and contextual meaning, and contrast between them. Therefore, it is crucial to make use of dictionaries to establish the fundamental connotation of a lexical element. Some scholars from the Amsterdam VU University have elaborated and adjusted the Metaphor Identification Process to some extent (Steen et al. 2010). Their method has minor differences, but basically it is the same. One distinction is that when making a decision whether a lexical unit is employed metaphorically, its history is not considered. Different studies have examined these two methods successfully (e.g. Steen et al. 2010). Still, the MIP does not show how to identify conceptual metaphors. In their studies, Steen $(1997,2009)$ and Semino (2008) discussed how to make the 'jump' from linguistic metaphor to conceptual metaphor. The studies of Steen (2008) suggest a five-step procedure that can be used for both linguistic metaphor and conceptual metaphor identification:

1) identifying metaphorical focus,

2) identifying metaphorical idea,

3) identifying metaphorical comparison,

4) identifying metaphorical analogy, and

5) identifying metaphorical mapping.

Semino (2008) claims that if the basic meaning and the contextual meaning of a metaphorical expression are understood in terms of an element of a topic domain and an element of vehicle domain respectively, it can help us to infer the underlying conceptual metaphor in conjunction with what is already known or common knowledge. We can also identify the conceptual metaphor underlying the metaphorical expression according to Steen's (2009) recommendation that if metaphor in discourse can be explained by means of an underlying cross-domain mapping in conceptual structure, then it should be possible to move from the linguistic forms in the text to the conceptual structures that capture their meaning in some ordered fashion.

The general framework of the conceptual metaphor theory (CMT) is adopted which provides a systematic way of deducing conceptual representations and organization from linguistic expressions. The underlying theoretical 
assumption of CMT allows us to link metaphorical expressions to underlying conceptual metaphors and hence the position of the conceptual mappings between the two conceptual domains. In conclusion, while Metaphor Identification Process (MIP) offers a reliable, reproducible, unambiguous method to recognize metaphor in language, it is time-consuming.

\section{Method and Data Collection}

The data in this study, which are in the form of emotive metaphorical expressions were taken from the Persian novel which was translated into English. The novel that was selected as most appropriate is Suvashun, a "best seller" novel written by Simin Daneshvar, a female Iranian scholar, novelist, fiction writer, and translator who has rendered many books from English, German, Italian and Russian into Persian. This novel was selected since it is replete with figurative languages, including metaphorical expressions which were the emphasis of this study. Consequently, metaphor variations available in this Persian novel allow the researcher to provide clear examples for their considered schemes in the present study. This Persian novel has been translated by 17 translators into various languages including German (with the title of Drama der Trauer- Suvashun), Spanish (with the title of Suvashun), French, Japanese, Russian, Chinese and Turkish, as well as two English translations. As such the novel and its translations serve as an excellent source of data for the present investigation. In addition, the original Persian title has been reprinted over 20 times and has been in circulation among half a million readers, maintaining its popularity since its first publication almost forty years ago. There are two famous English translations of Suvashun, by Mohammad Reza Ghanoonparvar and Roxane Zand in 1990 and 1992, respectively, both Iranian and obviously familiar with the Persian language. It is expected that they would have certainly tried to explain the cultural concepts accurately. Mohammad Reza Ghanoonparvar retained the original name of the novel but Roxane Zand changed the title to "A Persian Requiem". (Safarnejad \& Imran, 2013, p. 196). The choice of the two English translations for this study is based on one crucial interesting difference between the two translators. Both are originally from Iran but now live outside Iran, and both are bilingual, and are more than familiar with Persian metaphorical expressions and cultural concepts. However, the two translators seem to have applied different strategies in their translation of the same novel. As mentioned earlier, Roxana Zand changed the original title of the novel to A Persian Requiem while Mohammad Reza Ghanoonparvar left it unchanged. Furthermore, Zand seemed to have had problems in translating some metaphorical expressions and cultural concepts and when she was unable to find equivalent expressions for them, she omitted them. In fact, hers is rendered rather like a free translation. In contrast, the translation by Mohammad Reza Ghanoonparvar seems to have treated the transporting of the Persian metaphorical expressions and cultural concepts into English quite differently.

In conducting the present research, first of all the Persian sentences were studied one by one to extract a metaphoric statement. The unit of analysis in this research began at the level of word or phrase and whenever necessary, the scope of analysis was expanded to the context of the situation. There were no completely reliable methods for identifying metaphors, as they are not always in the format of ' $\mathrm{X}$ ' is ' $\mathrm{Y}$ '. However, a group of scholars, called Peragglejaz Group proposed partially reliable and flexible method for identifying metaphorically used word, which can be used for the purpose of this research. Therefore, the data were recorded, classified and analyzed descriptively. In terms of the procedure of the data collection, in the first stage, the data were obtained by collecting the Persian metaphorical expressions from the aforementioned novel. In the second stage, the English equivalents of the expressions in the two English translations of the novel were identified. In addition, the emotive metaphorical expressions relating to happiness/sadness from the source text and two target texts were identified to infer conceptual metaphor from the metaphorical expressions. In resolving the semantics of the lexical unit, the researchers relied on the Dehkhoda Dictionary (1999) and Aryanpur Bilingual Dictionary (1986), as well as the Macmillan English Dictionary for Advanced Learners of American English (MED 2008). The translational equivalences of these metaphorical expressions, thus, were identified in the target texts. The conceptual metaphors of the translation were searched and compared with those of conceptual metaphors in the source text. Finally, based on the analysis of the metaphorical expressions and the conceptual metaphors in the source text and the target texts, flexible patterns were found through the examination of emotive metaphorical expressions translation to better help translators to overcome the ordinary barriers while conveying Persian metaphor into English. It is presumed that the result of the current study will present an adequate functional explanation to propose some broad metaphoric translation patterns. Within the current study, the cognitive equivalence concept is deemed helpful as well since the researchers look for such equivalence among emotive metaphorical expressions as conceptual structures between the English and Persian manuscripts. It is supposed that the Persian and English texts would share similar conceptualization of happiness and sadness at universal concepts, while differences may arise at especial metaphorical expressions due to cultural specificity. It is also 
assumed that similarities reflect a common conceptual ground that would be evident more in common expressions. These issues will be followed up in data analysis.

\section{Data Analysis and Discussion}

In order to analyze the data, metaphorical expressions from the aforementioned novel and their corresponding English translation were gathered, and the conceptual metaphor underling the metaphorical expressions were identified. Therefore, after analyzing the metaphorical expressions in the source text and the target text and grouping them into conceptual domains of happiness and sadness, a qualitative assessment pattern of translating metaphorical expressions degree of application was carried out to show the Persian and English texts' degree of convergence or divergence in conceptualizing happiness/sadness. The researchers identified a total of 50 metaphorical expressions in the source text. This number included 14 happiness metaphorical expressions and 36 sadness metaphorical expressions extracted from the Persian source text which have been translated in two target English texts. In this paper, because of the large number of metaphorical expressions, some cases of linguistic expressions were discussed to illustrate the similarities and differences of emotive metaphoric conceptualizations between the source culture and the target culture. Therefore, the analysis of some metaphorical expressions which were collected from the aforementioned texts is presented in two parts as below respectively.

\subsection{Discussion of Happiness Metaphorical Expressions}

According to the application of MIP, the researchers determined the lexical units which were the sources of metaphors in each of the expressions (these are in bold). The discussion below focuses on each example and its translations.

1. ST (Page22) : ابولقاسم خان مثل شاخ شمشاد تو آ مد

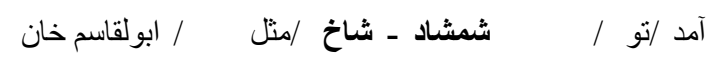

Transliteration: Abolgasem khan / mesl-e / shakh-e- shemshad / tu / amad

Abolgasem khan / like-of / branch-of- boxtree / in / come

Literal Translation: He like branch boxtree comes.

1a. He comes in straight as an arrow. (TT1 page 37)

1b. He walked in with a brisk air. (TT2 page 17)

2. ST (page174): حميد خان جاق و شنغول وارد شند

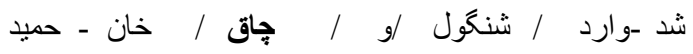

Transliteration: hamid khan / chagh / va / shangol/vared- shod

Hamid khan/ chubby / and / cheerful / enter- become

Literal translation: he entered with fat and cheerful.

2a. He entered with fat and joy. (TT1 page 186)

2b. He arrived. He looked plump and jolly. (TT2 page 160)

3. ST (page 125): هرمز از سرخوشى خنديد

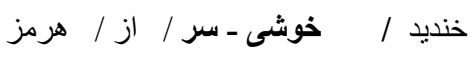

Transliteration: Hormoz / az / sar-khoshi / khandid

$$
\text { Hormoz / of / head -happy / laughed }
$$

Literal translation: Hormoz laughed of happy-headed.

3a. He was so happy. (TT1 page 138)

3b. He laughed uproariously. (TT2 page 115)

In (1), the compound words "shakhe-shemshad" (Lit: branch-boxtree) is considered as a potentially metaphorical item. The contextual meaning of "shakhe-shemshad" (Lit:branch-boxtree) in this context used to describe a happy person with upright posture. "shakhe-shemshad" is a common metaphor in Persian (Farhang-e Kenayat-e Sokhan, 2004). Therefore, this metaphorical expression is based upon our bodily experience. The most basic meaning of "shakhe-shemshad" (Lit: branch-boxtree) is the name of a tall tree with permanent and small shiny leaves. Its wood is hard and firm. In this context, there is a contrast between the first discussed contextual meaning of "shakhe-shemshad" and its basic meaning. The basic meaning of "shakhe-shemshad" is a kind of tree, while the contextual meaning indicates a metaphorical concept that expresses happiness in terms of upward 
posture. It can be concluded that there is a contrast between the contextual meaning and the basic meaning; consequently, "shakhe-shemshad" (Lit:branch-boxtree) is metaphorical. Considering the context of this story, seeing from the sentence Abolghasem Khan is compared to "shakhe-shemshad". The image of "shakhe shemshad" (Lit: boxtree) was chosen because of the characteristic of the "branch-boxtree" that is tall with green shiny leaves in all seasons of the year. Therefore, "shakhe shemshad" refers to the physical upright posture for a happy person with positive emotional state, while the opposite drooping posture is consistent with a negative one. In other words, this can be explained by Lakoff and Johnson's (1980) note that happiness corresponds to the physical experience of standing or walking upright, while depression corresponds to the physical experience of lying down when we are tired or depressed. Therefore, the conceptual metaphor underlying the expression "Abolgasem khan mesle shakhe shemshad vared shod" (Lit: He comes in like branch boxtree) is HAPPINESS IS UP.

The translations of the metaphorical expression in (1) are retrieved by aligning the source context and the target contexts and are represented below in (1a) and (1b):

1. Abolgasem khan / mesl-e / shakh-e-shemshad / tu / amad

Abolgasem khan / like-of / branch-of-boxtree / in / come

1a. He comes in straight as an arrow. (TT1 page 37)

1b. He walked in with a brisk air. (TT2 page 17)

The analysis of the English translation data follows the analysis of the Persian data and involves a sequence of MIP steps. In the target text (1a), the phrase "straight as an arrow" is considered as a potentially metaphorical item. In this context, the translator used a different phrase "straight as an arrow" instead of "shakhe shemshad" from the source text. According to NTC's American Idioms Dictionary (2000), "straight as an arrow" is defined "if used for something refer to very straight but if it is used for someone that means "honest or forthright". In this context, the contextual meaning of the phrase "he comes in straight as an arrow" is considered as "a movement very fast". The most basic meaning found in the Macmillan English Dictionary (2008) of the lexical unit "an arrow" is "a weapon in the form of a thin straight stick with a sharp point at one end and feathers at the other". In this context, there is a contrast between the contextual meaning and the basic meaning. Regarding the contextual meaning, "he comes in straight as an arrow", it expresses the movement of something that comes very fast. Therefore, the translator used new metaphor which has changed the meaning in the target text (1). In the instance above, "straight as an arrow" shows horizontal axes in the target text (1a) instead of upward or vertical space from the source metaphorical expression. In the Persian language, the expression "straight as an arrow" just connotes forthright when someone has quick movement. So, the concept of 'happiness is up' in the target language (1a) has changed.

In target text $2(1 \mathrm{~b})$, in this context, there is not a lexical unit with potentially metaphoric meaning. Considering the context of the target text $2(1 \mathrm{~b})$, the translator has changed the image and instead of "shakhe shemshad" has used "brisk air" which is not a metaphorical expression in this context. The translator in (1b) deletes the metaphor "shakhe shemshad" without trying to find an appropriate equivalent. According to the Macmillan English Dictionary (2008), "brisk" is defined as an adjective. This word is sometimes used for saying that the weather is fairly cold and a fairly strong wind is blowing. Therefore, in the context "He walked in with a brisk air", there is no hidden meaning that needs to be uncovered. In fact, the expression "He walked in with a brisk air" represents a non-metaphorical translation of the original metaphor in the source text. Consequently, the analysis of this example shows that both translators used different expressions in English.

In (2), "hamid khan chagh va shangol vared- shod" (lit: he entered with fat and cheerful), the lexical unit "chagh" (Lit:fat) is considered as a potentially metaphorical item. In this context, "chagh" and "shanghol" are synonymous. The lexical unit "chagh" in the context of the expression refers to "joyful and lively person". According to Aryanpur Bilingual Dictionary (1986), the basic (non-metaphoric) meaning of "chagh" is "chubby that slightly overweight". When we compare the contextual meaning and the basic meaning of "chagh", we notice that the contextual meaning contrasts with the basic meaning. We understand that the basic meaning of "chagh" refers to "a person is chubby". This meaning is in contrast with the meaning of "chagh" in the expression where the context is one where refer to the behavioral a person"he entered with full of life" to denote an emotion, in this context, happiness. The expression "hamid khan "chagh va shangol vared shod" (Lit: he entered with fat and cheerful) is used metaphorically to denote vivacity and liveliness as a source of happiness. Happiness and liveliness are really connected, and that one's level of happiness really can impact the level of one's vitality. In terms of human physiology, when a person feels well, he is also healthy, and then he is well placed to experience the exhilaration, joy and happiness. Hence, the conceptual metaphor underlying the 


\section{expression is HAPPINESS IS VITALITY.}

The translations of (2) are provided in (2a) and (2b).

\section{Hamid khan/chagh / va / shangol / vared- shod}

Hamid khan/ chubby/ and / cheerful / enter- become

2a. He entered with fat and joy. (TT1 page 186)

2b. He arrived. He looked plump and jolly. (TT2 page 160)

The English translation in TT1 and TT2 represents a non-metaphorical translation of the original metaphorical expression "hamid khan chagh va shangol vared shod" (Lit: he entered fat and cheerful. In the translation of this sentence, the translator mentioned the exact metaphor as the original, but since the target reader is not familiar with the concept behind the above metaphor, without referring to the contextual meaning of the source context, the translation may be acceptable; however, since the context given in the source context is about vitality and liveliness, the context factor should be taken into consideration when the translator translates the word "chagh" (fat). It is actually not referring to vitality and liveliness. It is obvious that the translator in (2a) matches only lexical items between the source language and the target language. To elaborate further, the word "chagh" has been translated into "fat" and this is the basic meaning of the word "chagh". The literal translation of the metaphorical expression "hamid khan chagh va shangol vared shod" (He entered with fat and joy) failed to convey the meaning of vitality. Hence, we can realize that the original metaphor does not fit in the target text due to conceptualizations of happiness intolerance in the target text 1 (2a).

In target text 2, the source metaphorical expression "hamid khan chagh va shangol vared shod" is translated into "He arrived. He looked plump and jolly" which is not a metaphorical item in this case. To illustrate more, the meaning of the original metaphor was completely changed in the target text 2 (2b). The expression can be understood literally. There is no hidden meaning that needs to be examined in order to understand the phrase itself. To explain more, during translating of the source metaphorical expression into English, the translator changed the linguistic structure with misinterpretation of the source metaphorical expression. However, the concept of "chagh va shangol" (vitality) does not exist in English and is vague for English readers. To illustrate more, both translators have translated the expression too literally in English.

In (3), the lexical unit "sarkhoshi" (Lit: happy-headed) is considered as a potentially metaphorical item. The contextual meaning of "sar-khoshi" (Lit: happy-headed) refers to full of high-spirited delight. Considering the context of the story, "happy-head" refers to a character of the story that is overjoyed. According to Dehkhoda Persian Dictionary (1995), "sarkhoshi" is a happy feeling that can be embodied in the head. It is important to notice that the head is conceptualized as the site of emotion in this context. The most basic meaning of "sar" (Lit: head), according to the concise Persian English Dictionary (1986), refers to the upper part of the human body, that contains the face and brains. The difference is found through contrasting the contextual meaning with the basic meaning. The above example shows that in the Persian metaphorical expression, the head can also be the container of emotion. It is maybe assumed that the head, instead of being a container for feelings, is seen as a container for opinions that carry particular types of emotions; but, indeed, the head indirectly shows its role as a container for emotions. Also, we should pay attention to this issue that while the head is regarded as a container for opinions, the heart is observed to take the same role for feelings. Nevertheless, it can be said that "His head is full of joy" is considered to be the prototypical emotion because this metaphoric expression is easier to be conceptualized as opinion than happiness. Although the head is known as the source of reason (see Kovecses, 1990: 179), a couple of metaphorical expressions depict it as a container for emotional beliefs. In this regard, some examples can be found in the following: "Her head was full of happy cares". It can be seen that the head is used as a metonymy for a person that can even reflect emotions, which are always connected with the heart. Besides, when a person is happy, his heart becomes containers for emotions. Thus, the head can be felt as containing the same type of emotions as the heart. Hence, the conceptual metaphor "HAPPINESS IS A FLUID IN A CONTAINER" is also instantiated by the linguistic expression "Hormoz az sar-khoshi khandid" in the source context (3).

Example (3) in our data is translated as (3a) in TT1 and (3b) in TT2.

3. Hormoz / az / sar-khoshi / khandid

Hormoz / of / head-happy / laughed

3a. He was so happy. (TT1 page 138)

3b. He laughed uproariously. (TT2 page 115) 
The English translation in (3a) represents non-metaphorical rendition of original metaphorical expression. The source metaphoric item "sarkhoshi" is translated into "very happy" in TT1. The translator merely conveys the literal meaning of the source metaphorical expression. Similarly, in TT2, the translator has used non-metaphor expression "He laughed uproariously" to describe happiness. In this case, there is no hidden meaning that needs to be uncovered. Consequently, the English translation does not involve an original expression.

\subsection{Discussion of Sadness Metaphorical Expressions}

The analysis of sadness expression follows the analysis of the happiness expressions and involves a sequence of steps. In this section, the metaphorical expression relates to sadness are presented as below:

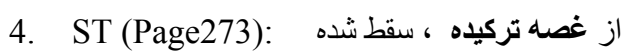

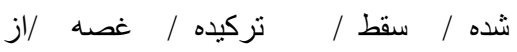

Transliteration: az/ghoseh/tarakideh/saghat / shodeh

$$
\mathrm{He} / \text { grief / explode / died / became }
$$

Literal Translation: He explodes of grief and died.

4a. he collapsed and died of grief.. (TT1 page 37)

$4 \mathrm{~b}$. he had died of grief right there. (TT2 page 17)

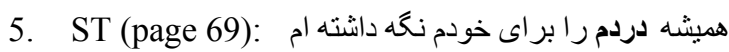

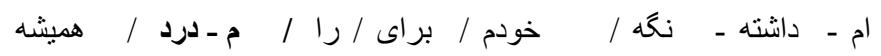

Transliteration: homes / dared- am / Ra / barley / khodam / negah- dashte- am

Always /Pain- my / Do/ for / myself / hold - has- she

Literal translation: Always holding my pain for myself.

5a. I have always kept my pain to myself. (TT1 page 82)

5b. I have always kept my grief to myself. (TT2 page 61)

6. ST. (page100): با دلمردگى شروع كرد /

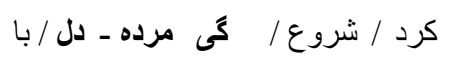

Transliteration: ba/ del- morde-gi / shoro / kard

$$
\text { with / heart- dead-she/ start / do }
$$

Literal translation: she began with heart dead.

6a. dejectedly she began. (TT1 page 114)

6b. downhearted she began. (TT1 page92)

In (4), the lexical unit "tarakide" (Lit: explode) is considered as a potentially metaphorical item. In this context, the contextual meaning of the lexical unit "tarakide" (Lit: explode) refers to emotional suffering. The basic (non-metaphoric) meaning of "tarakide", according to Aryanpur Bilingual Dictionary (1986), is "something that burst with a lot of force. When we compare the contextual meaning and the basic meaning of "tarakide", we notice that the contextual meaning contrasts with the basic meaning. We understand the basic meaning of "tarakide" in Persian as something that burst with physical force. This meaning is in contrast to the meaning of "az ghose tarakide" in (4) where the context is one where someone explodes with strong emotion, in this case, sadness. Hence, the lexical unit "tarakid" (Lit: explode) is marked as metaphorical item. The expression "ou az ghose tarakideh" (Lit: He explodes of sorrow) is used metaphorically to denote a person who has an overwhelming feeling of sadness. Considering the context of the story, the expression "ou az ghose tarakideh" refer to one of the characters of the story who had experienced heightened period of sadness after the death of a loved one. The author of the source text intends to say that the resultant loss of someone is almost never a singular event. In fact, heightened period of sadness can cause someone to lose all the hopes, expectations about dreams and life. It is possible to say that the intensity of negative emotion could affect inside the body negatively. So, in the Persian metaphoric expression "ou az ghose tarakideh" (Lit: He explodes of grief), the intensity of sad feeling is shown as fluid located inside the container (body) that could not be controlled by the subject which may be harmful. The negative metaphorical sense of sadness may conceptualize to people experience a deep sense of loss. There may be moments when people find themselves at a loss for words, weeping, or bursting uncontrollably into tears. When grief is so overwhelming and suffering, it can affect on health. Also, the immune 
system is closely related to our emotional state and can make a person extremely vulnerable to potentially fatal infections, and depression from grief can also be a terribly dangerous problem. Severe grief can even produce a physical condition like the shock from physical trauma. In shock, organ systems can shut down and grief could cause an elevated blood pressure that could cause a stroke or heart attack. Hence, the Persian metaphorical expression shows the conceptual metaphor of SADNESS IS THE BUILD UP OF PRESSUR IN A CONTAINER.

Example (4) in our data is translated as (4a) in TT1 and (4b) in TT2.

\section{4. az/ghoseh/tarakideh/saghat/ shodeh}

of/grief / explode / died / became

4a. he collapsed and died of grief. (TT1 page 37)

$4 \mathrm{~b}$. he had died of grief right there. (TT2 page 17)

In the translated version, there is not a lexical unit with potentially metaphoric meaning. The both translators delete the metaphorical phrase "az ghose tarakideh" (Lit: explode of grief) without trying to find an appropriate equivalent. In fact, both translators used non-metaphor rendition of the source metaphorical expressions. The Persian expression "az ghose tarakideh" (Lit: He explodes of grief) is translated literally as "he collapsed and died of grief" in (4a) and "he had died of grief right there" in (4b). The image of "exploding with grief" does not exist in English. Therefore, in the above two English contexts, there is no hidden meaning that needs to be uncovered. We can see that the Persian metaphorical expression is paraphrased. The concept of sadness is translated into English literally, which causes the loss of the original metaphorical phrase of the source text. Consequently, the example above shows that the translation does not follow the source expression closely. Both translators literally translate the Persian expression and reproduce similar concept in the target language, because they feel that the English translation is comprehensible in the target culture and evokes the similar concept in the target language audience.

In (5), "hamishe dard-am ra baray-e khodam negah dashte-am" (Lit: Always holding my pain for myself), the compound "dard-negah-dashtan" (Lit: hold-pain) is considered as a potential metaphorical item. In this context, the contextual meaning of the lexical unit "dard" (Lit: pain) is "suffer painful emotion". Referring to the context of the story, the reader realizes that the author of the original text is talking about painful memories that can become heartache. Considering the concept of the story, one character of the story has painful experiences that occurred in the past, such as the loss of her family. The basic (non-metaphoric) meaning of "dard-negahdashtan" (Lit: hold-pain), according to Aryanpur Bilingual Dictionary (1986), is "bodily suffering in specifically psychical sense". So, in this context, the contextual meaning contrasts with the basic meaning. The expression "holding my pain for myself" is metaphorically used to denote mental suffering in this case sadness. The author skillfully connected one of the features of sadness to pain. The Persian expression reveals that when a person hides negative emotion and internalizes sadness, it may suffer under the pain. Sadness and pain are really connected. In terms of human physiology, when we are very sad, in fact, we are experiencing both physical and emotional pain because the mind, body and emotions are connected. Therefore, the expression reflects the conceptual metaphor SADNESS IS A BURDEN.

Example (5) in our data is translated as (5a) in TT1 and (5b) in TT2.

5. hamishe / dard- am / ra / baraye / khodam / negah-dashte- am

Always /Pain- my / Do/ for / myself / hold-have - I

5a. I have always kept my pain to myself. (TT1 page 82)

5b. I have always kept my grief to myself. (TT2 page 61)

In target text 1 (5.a), the compound "kept-pain" is considered as a potentially metaphorical item. In this context, the contextual meaning of the English expression refers to grief. In the target text1 (5.a), the English translation 'I have always kept my pain to myself" represents the exact metaphorical expression as the original "hamishe dard-am ra baray-e khodam negah dashte-am". The translator prefers to save the original expression in his translation and produces the same concept in the target language. Through choosing the lexical unit "kept-pain" in the target text (5.a), the translator has managed to maintain the meaning of the original lexical unit in the source text. The expression "keeping one's pain" in English is closely related to lexical unit "negahdashtan-dard" in Persian. However, the English translation represents that the translator utilized similar lexical and semantic features. Due to this fact, the translator was able to reproduce the same concept in his translation when he says "always kept my pain to myself". Furthermore, a similar conceptual comprehensible 
metaphor namely SADNESS IS A BURDEN is used in translation 1 (5.7a).

In target text 2 (5.b), the compound "kept-grief" in persian is considered as a potentially metaphorical item. It is obvious that the English expression "I have always kept my grief to myself" is not an exact literal translation of the original sentence in the source text. In this example, the translator uses different though related metaphorical expressions. To explain more, by using the word "grief" instead of "dard" (lit: pain), the translator finds the approximate corresponding lexical unit in the target language. Furthermore, the linguistic term is different, but the metaphorical thought process is similar. The translator saves the original metaphor with a slight variation in its format and reproduces the same image in the target language. The translator also does not directly address the grief as a pain. Hence, the conceptual metaphor SADNESS IS A BURDEN is also instantiated by the linguistic expression "I have always kept my grief to myself" in the target text 2 (5.b). Based upon the above examples, it can be concluded that both the original context and the translation contexts indicate similar concept of sadness. The universal metaphoric concept makes the translator's task easier in achieving a higher level of equivalence by finding the similar expression of the source text that already exists in the target texts.

In (6), the lexical unit "del-mordegi" (Lit: heart-dead) is considered as a potentially metaphorical item. "del-mordegi" is a compound noun in Persian made up of the morphemes del 'heart' and morde 'dead'. This compound noun as a whole literally glossed in English as "heart-dead". The contextual meaning of "del-mordegi" (Lit: heart-dead) in this context used to describe someone who is cheerless. The basic (non-metaphoric) meaning of "del" (Lit: heart), according to Aryanpur Bilingual Dictionary (1986), is as "heart, stomach, abdomen, belly, guts" and basic meaning of "morde" is "end of life". When we compare the contextual meaning and the basic meaning of "delmorde", we notice that the contextual meaning contrasts with the basic meaning. Hence, the lexical unit "del-mordegi" (Lit: heart-dead) is marked as metaphorical item. The metaphorical expression reflects conceptualization of the heart as the center of personality mood. This shows that whenever a person is sad, his heart is sad too. Persian uses many heart metaphorical expressions with emotional meaning of sadness. In fact, the metaphorical expression "delmorde" (Lit: dead heart) is in contrast to "delzende" (Lit: living heart) in Persian language. It is to be noted that in Persian culture, the expression "delzende" (Lit: living heart) is used for someone who is lively and cheerful, while, the expression "delmorde" (Lit: dead heart) refers to lack of vitality and living. Therefore, the expression reflects the conceptual metaphor SADNESS IS LACK OF VITALITY.

Example (6) in our data is translated as (6a) in TT1 and (6b) in TT2.

\section{6. ba/ del- morde-gi / shoro / kard}

with / heart- dead- she / start / did

6a. dejectedly she began. (TT1 page 114)

6b. downhearted she began. (TT1 page 92)

The English translation in (6a) represents non-metaphorical rendition of original metaphorical expression. The source metaphoric item "demordegi" (Lit: dead heart) is translated into "dejectedly" in TT1. In this context, contextual meaning and the basic meaning are similar. In fact, there is no contrast between the contextual meaning and the basic meaning. In this context, there is no hidden meaning that needs to be uncovered. Macmillan English Dictionary translates "dejected" as "someone who has lost all their hope or enthusiasm, especially because they have failed at something". Consequently, the translator merely conveys the literal meaning of the source metaphorical expression.

In target text 2 (6.b), the lexical unit "downhearted" is considered as a potentially metaphorical item. In this context, the original metaphorical expression "delmorde" (Lit: dead heart) does not exist in (6b) but is conceptually comprehensible. To explain more, by using the word "downhearted" instead of "delmordegi" (lit: dead heart), the translator preserved the concept of the original expressiveness. In the case of (6b), there is a shift in the metaphorical expression as well as the conceptual metaphor. In this context, the contextual meaning of "downhearted" refers to someone who is in low spirits. Thus, the conceptualization of sadness in the ST to TT2 is different. It leads us to conclude that the conceptual metaphor in the translation is SADNESS IS DOWN.

\section{Conclusion}

The present study has made an attempt to investigate metaphor translation using a cognitive approach to metaphor. Cultural differences between the source language and the target language, and between the source culture and the target culture, have often been mentioned as a problem for the translators of metaphorical expressions at the linguistic level and conceptual level. The above discussion of metaphorical expressions made it clear that the source culture and the target culture sometimes employ the same metaphorical mapping 
condition from the same source domain, and sometimes the source language and the target language reflect different conceptual metaphors from the same source domain. It should be noted that sometimes cultural lexical units are difficult to translate. This is due to this fact that some lexical units involve a cultural background and cultural knowledge. The analysis of emotive metaphorical expressions between two different languages showed that literal translation cannot completely provide the meaning of the cultural lexical units because they have a different semantic range in the source and the target languages (as presented in the case of 1.2.3.4 SL). Based upon the analysis of data in this study, we concluded some cases which have been identified as below:

1) The loss of metaphorical meaning of the source language to the target language can be conveyed by many cultural background lexical units. For example, it has been shown by the Persian word "chagh" (lit: chubby) in case of $2 \mathrm{ST}$.

2) A cultural word makes hard task the translation of metaphorical expression from one language to another language, as shown by the Persian lexical units "shakhe shemshad" (lit: boxtree) in the case 1.ST.

However, it should be considered that, sometimes the equivalent metaphors have identical linguistic forms and identical conceptual mapping; sometimes they have different linguistic forms and identical conceptual mapping, and sometimes they have different linguistic forms and equivalent conceptual mapping with the same metaphorical meaning. Consequently, based upon the cognitive view on metaphor translation, the following strategies can be used by the translators in translation of the source metaphorical expression:

1) Translation of the source metaphorical expression to the equivalent target language metaphor (As shown in cases $5 a, 5 b)$.

2) Translation of the source metaphorical expression to non-metaphor in the target language (As shown in cases 1b, 2a, 2b, 3a, 3b, 4a, 4b, 6a).

3) Mistranslation (As shown in cases 2a, 2b).

4) Literal translation was the very common strategy applied by two translators in translating Persian metaphors into English.

All in all, the analysis of the collected data showed that there is a mismatch between cognitive mapping in the two languages, i.e. cognitive mappings of concepts are different rather than similar in Persian and English due to different models of conceptualizing experiences in each culture. English and Persian cultures cover different realms for cognitive mapping of the same idea. The results show that there is some kind of equivalence between Persian and English in translating metaphorical expressions of happiness and sadness to some extent. Also Deignan and Potter (2004) state that some variations in the linguistic metaphors are due to the differences in the surface realization of some conceptual metaphors in linguistic associations. The reason for this difference in the translation process is that it is the search for another conceptual mapping (another cognitive domain) which causes delay, uncertainty and difficulty in the translation of different domain metaphors. According to Maalej (2004), the more the two cultures conceptualize an experience in a similar fashion, the more similar mapping applies; and the more they conceptualize an experience differently, the more different mapping applies. So different conceptual mapping between metaphors show that the two languages conceptualize emotions in different ways.

\section{References}

Al-hasnawi, A. R. (2007). A Cognitive Approach to Translating Metaphors. Cultural Aspects, 11(3). Retrieved from http://www.accurapid.com/journal/41metaphor.htm.

Al-Zoubi Mohammad, N., Mohammed, A-A., \& Al-Hasnawi Ali, R. (2007). Cogno-Cultural Issues in Translating Metaphors. Perspectives: Studies in Translatology, 14(3), 230-239.

Anvary, H. (2004). Farhang-e Kenayat-e Sokhan. Tehran, Iran: Sokhan.

Aryanpur Kashani, A. M. (1986). Persian-English Dictionary.

Catford, J. C. (1965). A Linguistic Theory of Translation. London: Oxford University Press.

Dagut, M. (1976, January 1). Can 'Metaphor' Be Translated? Babel, 22(1), 21-33. http://dx.doi.org/10.1075/babel.22.1.05dag

Dagut, M. (1987). More about the Translatability of Metaphor. Babel, 33, 77-82. http://dx.doi.org/10.1075/babel.33.2.06dag

Daneshvar, S. (1969). Savushun. Tehran: Kharazmi. 
Deignan, A., \& Potter, L. (2004). A corpus study of metaphors and metonyms in English and Italian. Journal of pragmatics, 36, 1231-1252. http://dx.doi.org/10.1016/j.pragma.2003.10.010

Dobrzyńska, T. (1995). Translating Metaphor: Problems of Meaning. Journal of Pragmatics, 24(6), 595-604. http://dx.doi.org/10.1016/0378-2166(95)00022-K

Ghanoonparvar, M. R. (1991). Savushun: A Novel about Modern Iran (Persian Classics). Mage Pub, Washington, D. C.

Group, P. (2007, January). MIP: A Method for Identifying Metaphorically Used Words in Discourse. Metaphor and Symbol, 22(1), 1-39.

Hiraga, M. K. (1991). Metaphor and Comparative Cultures. Cross-Cultural Communication: East and West, 3, 149-166.

Iranmanesh, A., \& Kulwindr Kaur, G. S. (2010). A Cognitive Approach and Translation Strategies Used in the Subtitling of Metaphors in Three American Movies. Retrieved from http://english.um.edu.my

Kövecses, Z. (1990). Emotion Concepts. Berlin and New York: Springer Verlag. http://dx.doi.org/10.1007/978-1-4612-3312-1

Kovecses, Z. (2005). Metaphor in Culture. Universality and Variation. Cambridge: Cambridge University Press. http://dx.doi.org/10.1017/CBO9780511614408

Lakoff, G., \& Johnson, M. (1980). Metaphors We Live By. Chicago: The University of Chicago Press.

Maalej, Z. (2002). Book Review: Metaphor and Metonymy at the Crossroads. A Cognitive Perspective. Language and Literature, 11, 180-183. http://dx.doi.org/10.1177/096394700201100209

Maalej, Z. (2008). Translating Metaphor Between Unrelated Cultures: A Cognitive-pragmatic Perspective. Sayyab Translation Journal, 1, 60-81.

Macmillan English Dictionary. (2008). For Advanced Learners of American English.

Mandelblit, N. (1995). The Cognitive View of Metaphor and Its Implication for Translation Theory. Maastricht: Maastricht University Press.

Nida, E. (1964). Toward A Science of Translation. Netherlands: Leiden.

Richard, A., \& Spears, P. H. D. (2000). NTC's American Idioms Dictionary. NTC publishing Group.

Schäffner, C. (2004). Metaphor and Translation: Some Implications of a Cognitive Approach. Journal of Pragmatics, 36(7), 1253-1269. http://dx.doi.org/10.1016/j.pragma.2003.10.012

Semino, E. (2008). Metaphor in Discourse. Cambridge: Cambridge University Press.

Snell-Hornby, M. (1988). Translation Studies (2nd ed.). Amsterdam, John Benjamins. http://dx.doi.org/10.1075/z.38

Steen, G. J. (1999). From Linguistic to Conceptual Metaphor in Five Steps. Metaphor in Cognitive Linguistics. Amsterdam: John Benjamins, 57-77. http://dx.doi.org/10.1075/cilt.175.05ste

Steen, G. J. (2009). From Linguistic to Conceptual Structure in Five Steps: Analyzing Metaphor in Poetry. Cognitive Poetics, 4, 765-796.

Tabakowska, E. (1993). Cognitive Linguistics and Poetics of Translation. Tübingen: Gunter Narr.

Van Den Broeck, R. (1981). The Limits of Translatability Exemplified by Metaphor Translation. Poetics Today, 2(4), 73-78. http://dx.doi.org/10.2307/1772487

Zand, R. (1992). A Persian Requiem. New York: G. Braziller.

\section{Copyrights}

Copyright for this article is retained by the author(s), with first publication rights granted to the journal.

This is an open-access article distributed under the terms and conditions of the Creative Commons Attribution license (http://creativecommons.org/licenses/by/3.0/). 